Research Article

\title{
Unit Experimental Study on Impact Crushing Rock of the Microcoring PDC Bit
}

\author{
Yingxin Yang $\mathbb{D}^{D}$, Dongdong Song $\mathbb{D}^{\mathbb{D}}$, Kuilin Huang, Haitao Ren, and Jian Zhou \\ School of Mechatronic Engineering, Southwest Petroleum University, Chengdu, China \\ Correspondence should be addressed to Yingxin Yang; yangyx36@163.com
}

Received 10 May 2019; Accepted 13 September 2019; Published 3 November 2019

Academic Editor: Salvatore Russo

Copyright ( 2019 Yingxin Yang et al. This is an open access article distributed under the Creative Commons Attribution License, which permits unrestricted use, distribution, and reproduction in any medium, provided the original work is properly cited.

In light of the situation of sliced or powdery cuttings produced by conventional PDC bit, it is found that they are not convenient for geological logging and stratification. The unit crushing experiment which contains two breaking forms (static-pressure breaking and impact) is conducted on core columns of sandstone, limestone, and granite. This experimental study showed the following: The breaking work ratio of the three core column types is much less than that of the flat rock. The height of the core column has little effect on the bit penetration rate, while core column diameter has greater effect on the bit penetration rate. The experimental results further indicate that a core column has been generated in the central area of the drill bit, which is beneficial to the volume breaking of the rock and can improve the rock-breaking efficiency of the drill bit. The microcoring bit is suitable for raising speed in soft, medium, and hard formations and is helpful for geological logging.

\section{Introduction}

Polycrystalline diamond compact (PDC) drill bits which adopt PDC as the cutting element deliver premium performance and durability (high ROP and life, as well as low drilling cost) in oil and gas drilling operations when drilling in soft, medium, and hard formations [1]. In oil exploration, the underground environment is complex. In order to verify the lithology, the corresponding tools, equipment, and drilling fluid properties must be cooperated to sample the rocks so as to determine the properties of the rocks in the formation and understand the mineral conditions of the formation $[2,3,4,5]$. Nevertheless, the debris generated during the drilling process of the conventional PDC bits is in the powder form and too small for formation identification; thus, different types of bits are required to meet the demand of full drilling and coring $[6,7,8]$. In view of the above conditions, a mass of related research has been carried out, and a mechanical microcoring PDC bit $[9,10]$ is put forward to push the broken core column outward depending on the mechanical force of the rock. Tercel Corporation has developed a microcoring bit which can form a coring groove instead of cutting teeth in the central area of the bit, which improves the ROP and effectively solves the problem of difficult coring $[11,12]$. The bit developed by TOTAL and Diamond Drilling Services Company can produce microcores, which can not only ensure high drilling efficiency in hard formation drilling but also obtain high-quality cuttings [13].

The breaking rules of core columns with different sizes are analyzed by unit breaking experiments, which are intended to select the most efficient rock-breaking method and core column type and provide the theoretical basis for the optimization design of the microcoring PDC bit to improve the rock-breaking efficiency of the bit and meet the accelerating demend for speed up drilling in multiple formations, which is helpful for geological logging.

\section{Unit Breaking Experiment}

There are two main methods for the microcoring bits to break the core column at the central area of the bit: staticpressure breaking and impact. Different breaking methods require different breaking energies, and the sizes of the generated debris are different as well. The diameter and height of the core column have a direct influence on the 
rock-breaking efficiency of the microcoring PDC bit, the sensitivity of which varies according to the breaking method. The unit breaking experiment of static-pressure breaking and impact is carried out on the core column of three types of lithology (sandstone, limestone, and granite) in different sizes.

\subsection{Static-Pressure Breaking Experiment}

2.1.1. Experimental Instruments and Rock Samples. The static-pressure breaking test instruments were a hydraulic testing apparatus, a displacement sensor, a pressure sensor, CZ1319 conical PDC teeth (including fixture), and a data acquisition system (DAQ system), as shown in Figure 1. Being connected to the teeth holder, the test teeth were fixed on the pressure sensor, which was connected to the hydraulic universal testing apparatus. Pressurization was realized by the upward movement of the testing apparatus, and the signal from the displacement sensor and pressure sensor is transferred to the computer for data acquisition through a strain gauge.

The rock samples (partly shown in Figure 2) for the static-load experiment were divided into 3 groups. The diameters of 3 types of the core column from the 1st group were $20 \mathrm{~mm}$, the heights of which were $20 \mathrm{~mm}, 25 \mathrm{~mm}$, $30 \mathrm{~mm}, 35 \mathrm{~mm}$, and $40 \mathrm{~mm}$. The heights of the sandstone core column from the 2 nd group were $30 \mathrm{~mm}$, the diameters of which were $20 \mathrm{~mm}, 25 \mathrm{~mm}, 30 \mathrm{~mm}, 35 \mathrm{~mm}$, and $40 \mathrm{~mm}$. The heights of the limestone core column from the 2 nd group were $30 \mathrm{~mm}$, the diameters of which were $20 \mathrm{~mm}$, $25 \mathrm{~mm}, 30 \mathrm{~mm}, 35 \mathrm{~mm}$, and $40 \mathrm{~mm}$. The heights of the granite core column from the 3 rd group were $30 \mathrm{~mm}$, the diameters of which were $20 \mathrm{~mm}, 25 \mathrm{~mm}, 30 \mathrm{~mm}, 35 \mathrm{~mm}$, and $40 \mathrm{~mm}$. Some rock samples of the core column are shown in Figure 2. The size of the 3 types of flat rock samples was $300 \mathrm{~mm} \times 100 \mathrm{~mm} \times 100 \mathrm{~mm}$, the lithology of which is shown in Table 1.

2.1.2. The Principle and Method of the Experiment. The rock sample was placed in the center of the testing machine platform, and the position of the rock sample was adjusted so that the cusp of the conical PDC tooth was aligned with the centerline of the rock sample. The height of the test platform was adjusted to make the tooth tip to be about $1 \mathrm{~mm}$ above the platform, the DAQ system was cleared and sampled, and then the rock sample was slowly loaded (Figure 3), until the rock sample was broken and the pressure load was unloaded; the experimental data were recorded and displayed by the DAQ system. The broken rock debris was collected, and an experiment was completed; each experiment was repeated at least 3 times.

\subsection{Impact Experiment}

2.2.1. Experimental Instruments and Rock Samples. The experiment instruments for the impact test were the DIT152Z full-automatic drop hammer test apparatus and the CZ1319 conical PDC teeth and fixture (Figure 4).

The rock samples for the impact experiment were divided into 3 groups, and the diameters of 3 types of the core

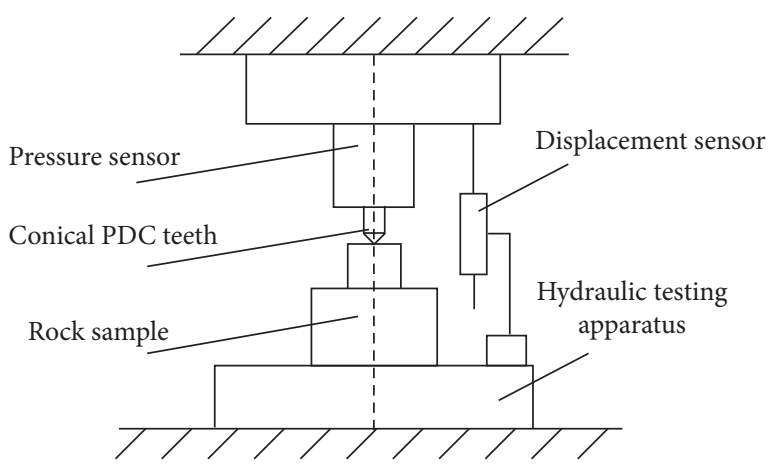

Figure 1: Schematic graph for the static-pressure breaking experiment (revised from [14]).

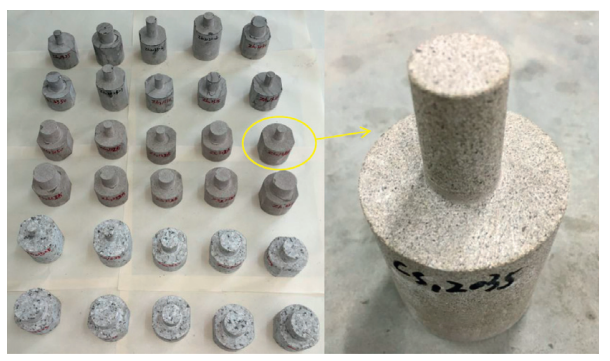

Figure 2: Core columns (partial).

column from the 1st group were $20 \mathrm{~mm}$, the heights of which were $20 \mathrm{~mm}, 25 \mathrm{~mm}, 30 \mathrm{~mm}, 35 \mathrm{~mm}, 40 \mathrm{~mm}$, and $50 \mathrm{~mm}$. The heights of the sandstone core column from the 2 group were $25 \mathrm{~mm}$, the diameters of which were $15 \mathrm{~mm}, 20 \mathrm{~mm}$, $25 \mathrm{~mm}, 30 \mathrm{~mm}, 35 \mathrm{~mm}$, and $40 \mathrm{~mm}$. The heights of the limestone core column from the 2 nd group were $30 \mathrm{~mm}$, the diameters of which were $20 \mathrm{~mm}, 25 \mathrm{~mm}, 30 \mathrm{~mm}, 35 \mathrm{~mm}$, and $40 \mathrm{~mm}$. The heights of the granite core column from the $3 \mathrm{rd}$ group were $25 \mathrm{~mm}$, the diameters of which were $15 \mathrm{~mm}$, $20 \mathrm{~mm}, 25 \mathrm{~mm}, 30 \mathrm{~mm}, 35 \mathrm{~mm}$, and $40 \mathrm{~mm}$. The size of the 3 types of flat rock samples was $300 \mathrm{~mm} \times 100 \mathrm{~mm} \times 100 \mathrm{~mm}$.

2.2.2. The Principle and Method of the Experiment. The rock sample was placed on the apparatus platform, and the conical teeth were connected to the drop hammer through a fixture. By moving the position of the rock sample, the axis of the cone was leveled with the axis of the rock. Adjusting the height of the drop hammer, the tooth tip is made to come into contact with the rock. Then, the impact energy was set, and the hammer body was automatically lifted to a certain height (Figure 5). The hammer was released at rest, causing the hammer body to fall freely and impact the rock sample; then, the drop hammer was reset to a certain height. The rock fragments were collected, and impact experiment was completed; each experiment was repeated at least 3 times.

\section{Criteria of Rock Breaking}

Nowadays, there are many evaluation criteria for the rockbreaking mechanism because of different evaluation indexes that are referred to, so a targeted solution is required 
TABle 1: Primary physical parameters of 3 rock samples.

\begin{tabular}{|c|c|c|c|c|c|c|}
\hline Rock & $\begin{array}{c}\text { Uniaxial compressive } \\
\text { strength }(\mathrm{MPa})\end{array}$ & $\begin{array}{l}\text { Shear strength } \\
(\mathrm{MPa})\end{array}$ & $\begin{array}{c}\text { Internal friction } \\
\text { angle }\left({ }^{\circ}\right)\end{array}$ & $\begin{array}{c}\text { Elastic modulus } \\
(\mathrm{GPa})\end{array}$ & $\begin{array}{l}\text { Poisson's } \\
\text { ratio }\end{array}$ & $\begin{array}{l}\text { Density } \\
\left(\mathrm{g} / \mathrm{cm}^{3}\right)\end{array}$ \\
\hline Sandstone & 67.548 & 13.56 & 38.03 & 11.54 & 0.062 & 2.42 \\
\hline Limestone & 105.951 & 17.72 & 43.62 & 31.2 & 0.171 & 2.46 \\
\hline Granite & 126.519 & 13.7 & 45.29 & 31.78 & 0.118 & 2.73 \\
\hline
\end{tabular}

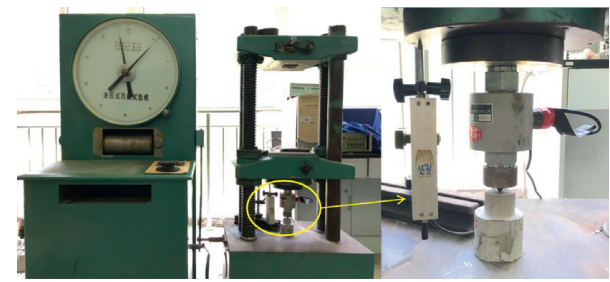

Figure 3: Process graph for the static-pressure breaking experiment.

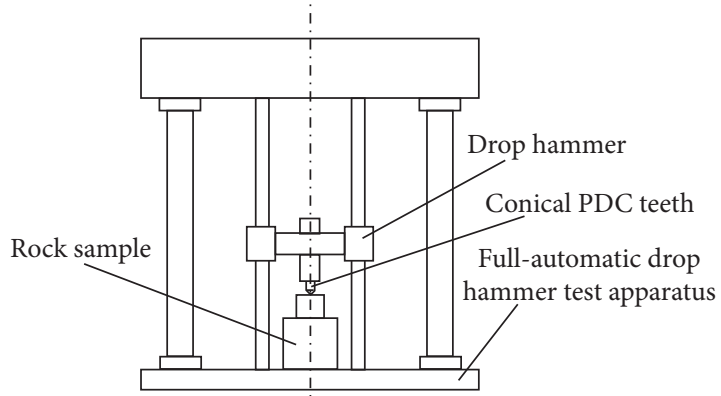

FIGURE 4: Schematic graph for the impact experiment.

according to different rock-breaking mechanisms. Two frequently used evaluation criteria for the rock-breaking mechanism are introduced [6].

3.1. Breaking Work Ratio per Unit Volume. The breaking work ratio per unit volume is defined as follows:

$$
W=\int_{0}^{h_{\max }} f(h) \mathrm{d} h,
$$

where $W$ is the work done by a single tooth during a period (from just contacting the rock to just breaking the rock) under the action of loading and $f(h)$ is the function of the load $F$ with respect to the depth (penetration depth of the tooth) $h$. Both $W$ and $h$ are calculated from the load-displacement curve.

$A_{\mathrm{V}}$ is a criterion for the breaking efficiency of the tooth, which is calculated by using the following equation:

$$
A_{\mathrm{V}}=\frac{W}{V},
$$

where $V$ is the volume of the breaking hole and $A_{\mathrm{V}}$ is the breaking work ratio per unit volume.

It is concluded that the smaller the $A_{\mathrm{V}}$, the higher the rock-breaking efficiency and vice versa, which is a good quantitative criterion for rock-breaking efficiency.
3.2. Rock-Breaking Efficiency Judged by Debris Granularity. Rittinger's surface theory [15] suggests that the physical and mechanical properties of rock have not changed before and after rock breaking. The difference is that the fragmentation (rock surface area) has changed. The new surface theory suggests that most of the work done by breaking the rock is used to form the new surface. The original fragmentation of the rock is $D$ and becomes $d$ after breaking. The surface area of a unit volume of the rock is directly proportional to the value of $(1 / d)-(1 / D)$. Thus, the breaking work ratio of the broken rock is defined as follows:

$$
A_{\mathrm{V}}=K_{\mathrm{r}}\left(\frac{1}{d}-\frac{1}{D}\right)
$$

Where $K_{\mathrm{r}}$ is a constant related to mechanical properties and breaking methods of rocks.

Generally, the size of average granularity is used to represent the fragmentation degree of the broken rock, which is defined as follows:

$$
d_{e}=\frac{\sum\left(r_{i} d_{i}\right)}{\sum r_{i}}
$$

where $d_{i}$ is the size of a certain granularity and $r_{i}$ is the percentage of the granularity.

When the size of the broken rock sample is too large, and $d$ of the debris (generated by breaking) is very small, $1 / D$ is quite smaller than $1 / d$ :

$$
A_{\mathrm{V}}=K_{\mathrm{r}} \frac{1}{d} .
$$

Equation (5) shows that the breaking work ratio is inversely proportional to the size of the debris; namely, the larger the cutting size, the smaller the breaking work ratio and the higher the rock-breaking efficiency.

\section{Results and Analysis of Unit Breaking Experiment}

4.1. Results and Analysis of Static-Pressure Breaking. In the static-pressure breaking experiment, tiny debris was generated directly below the teeth, which forms a compacting rock core with the increase of loading. The compacting rock core is the storage and transmission of the load energy, just as pushing a wedge into the rock. With the increase of loading, the tensile stress between the core and the ambient rocks becomes larger and larger until it exceeds the tensile strength of the rock, which causes the cracks to extend to the surface along the direction of maximum tensile stress and break the rock. During the extending process of the cracks, the tensile stress generated by the teeth when penetrating into the rock will gradually offset the internal stress of the 


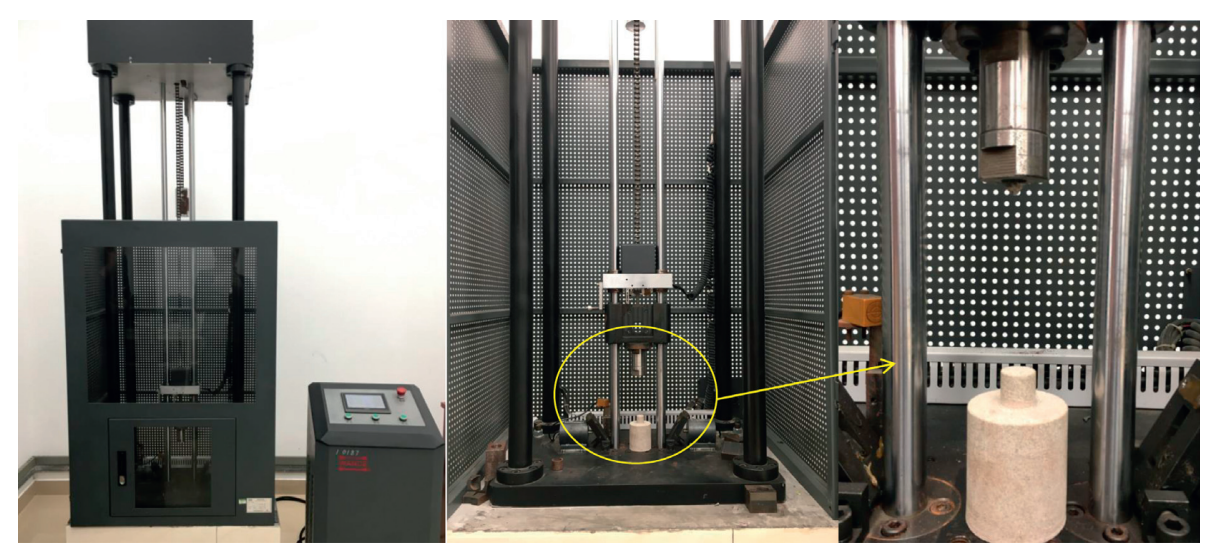

FIgURE 5: Process graph for the impact experiment.

rock and prevent cracks from extending to the deep. Thus, the rock sample will be broken only when the shallow cracks extend to the free surface of the core column (Figure 6). There are no constraints around the core column when the teeth are penetrating into the rock; thus, the cracks are able to extend to the base of the core column and break it on reaching the free surface of the core column (Figure 7). Compared to the flat rock samples, the debris generated by the core column is bigger (Figure 8) and so is the breaking volume; by contrast, the breaking energy is smaller.

The breaking work ratio is an important index to measure the breaking efficiency of the teeth, and the breaking work ratio of rock samples of the core column and flat rock with different lithology is obtained by calculation (Figure 9). The breaking work ratio of flat sandstone is $112.5 \mathrm{~J} / \mathrm{cm}^{3}$, while the maximum breaking work ratio of the sandstone core column is $0.6 \mathrm{~J} / \mathrm{cm}^{3}$, which is only $0.5 \%$ that of flat rock samples. The breaking work ratio of flat limestone is $90.1 \mathrm{~J} / \mathrm{cm}^{3}$, while the maximum breaking work ratio of the limestone core is $0.65 \mathrm{~J} / \mathrm{cm}^{3}$, which is only $0.7 \%$ that of the flat rock sample. The breaking work ratio of flat granite is $186.9 \mathrm{~J} / \mathrm{cm}^{3}$, while the maximum breaking work ratio of the core of granite is $0.95 \mathrm{~J} / \mathrm{cm}^{3}$, just $0.51 \%$ that of flat rock. It can be concluded that the breaking work ratio of the core column is much smaller than that of the flat rock sample, and the sensitivity of 3 types of rock to the shape of the rock sample is different: sandstone is the most sensitive, followed by granite and limestone.

It can be concluded from Figure 10(a) that, for the core column of the same height and with a diameter of $20 \mathrm{~mm}$, the breaking work ratio of granite is higher than that of sandstone and limestone, and with the change of height of the core column, the breaking work ratio of granite and sandstone undergoes a minor change, while there is a slight fluctuation in the breaking work ratio of the limestone. Granite, sandstone, and limestone have the minimum breaking work ratio when the heights of the core column are $35 \mathrm{~mm}, 35 \mathrm{~mm}$, and $30 \mathrm{~mm}$, respectively. Figure $10(\mathrm{~b})$ indicates that as the diameter of the rock pillar changes, the breaking work ratio of sandstone undergoes a minor change, which is the minimum when the diameter of the core column is $35 \mathrm{~mm}$, while there is a fluctuation in the breaking work ratio of limestone and granite, which is the minimum when the diameters of core columns are $30 \mathrm{~mm}$ and $20 \mathrm{~mm}$, respectively.

4.2. Results and Analysis of Impact. The teeth penetrate the rock under the impact load, and there are surface cracks and minor debris in the contact area. Beneath the debris, an approximate hemispherical shear compaction area (compacting rock core) is formed. A broken zone will be generated beneath the core, and there are three main cracks outside the broken zone [16]: lateral cracks, radial cracks, and intermediate cracks (Figure 11). Lateral cracks usually appear and expand during unloading, radial cracks appear during either loading or unloading, and intermediate cracks appear during the loading process and elastic recovery can occur to some of the cracks during unloading. The lateral cracks are parallel to the surface of the rock sample, which is generally round- or cake-shaped, and mostly appear near the compact rock core or extend to the free surface by the bifurcation of Hertz cracks, most of which will extend to the surface of the rock sample, causing the surface of a flat rock to fall off or collapse. With the increase of impact force, the compact rock core is crushed, and the crushed body cracks outward, generating a crater (Figure 12). When the teeth impact the core column, the impact energy generated by the core column is transferred downward along the axis of the core column because of the nonexistent constraint around the core column, causing the expansion direction of the radial cracks inside the rock sample to turn down, until they intersect with the surface of the core column, resulting in a saltatory fracture (Figure 13) to the core column; then, the ROP increases greatly, and the debris becomes larger (Figure 14).

Figure 15 shows that the breaking work ratio of the flat sandstone is $350 \mathrm{~J} / \mathrm{cm}^{3}$ and that the maximum breaking work ratio of the core column of the sandstone is $6.8 \mathrm{~J} / \mathrm{cm}^{3}$, only $2 \%$ that of the flat rock sample. The breaking work ratio of the flat limestone is $253 \mathrm{~J} / \mathrm{cm}^{3}$, and the maximum breaking work ratio of the core column of the limestone is $8 \mathrm{~J} / \mathrm{cm}^{3}$, only $3.2 \%$ that of the flat rock sample. The breaking work ratio of the flat granite is $529 \mathrm{~J} / \mathrm{cm}^{3}$, and the maximum breaking work ratio of the core column of granite is $5.6 \mathrm{~J} /$ $\mathrm{cm}^{3}$, only $1.1 \%$ that of the flat rock sample. It can be 


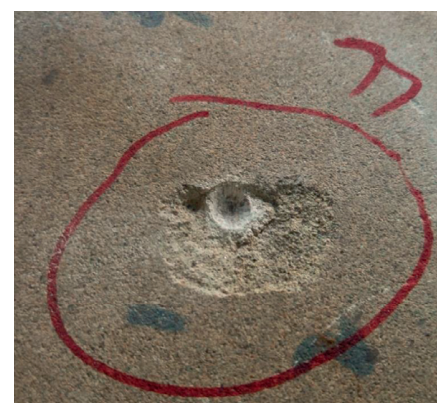

(a)

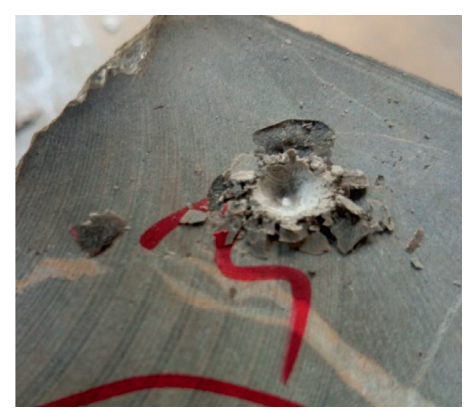

(b)

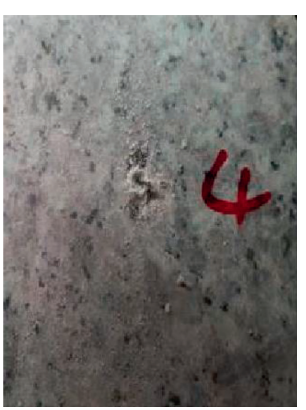

(c)

Figure 6: Breaking holes and debris of the flat samples and core columns under static-pressure breaking: (a) sandstone; (b) limestone; (c) granite (reproduced from [14]).

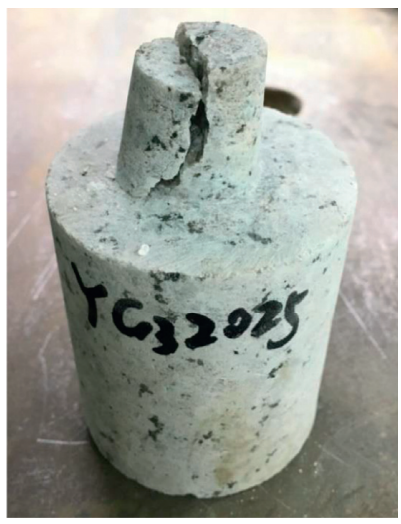

(a)

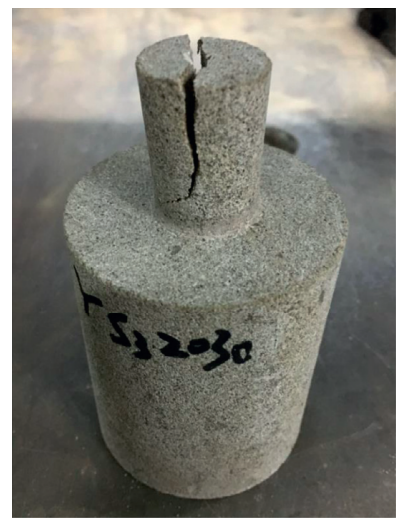

(b)

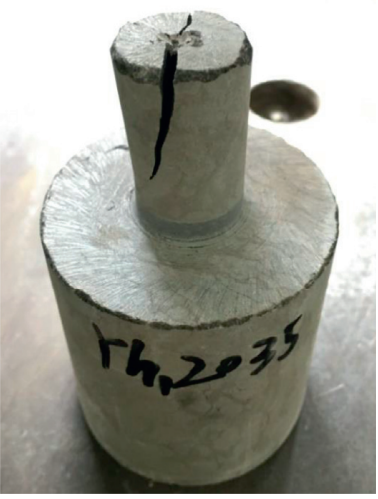

(c)

Figure 7: Broken core columns under static-pressure breaking: (a) sandstone; (b) limestone; (c) granite.

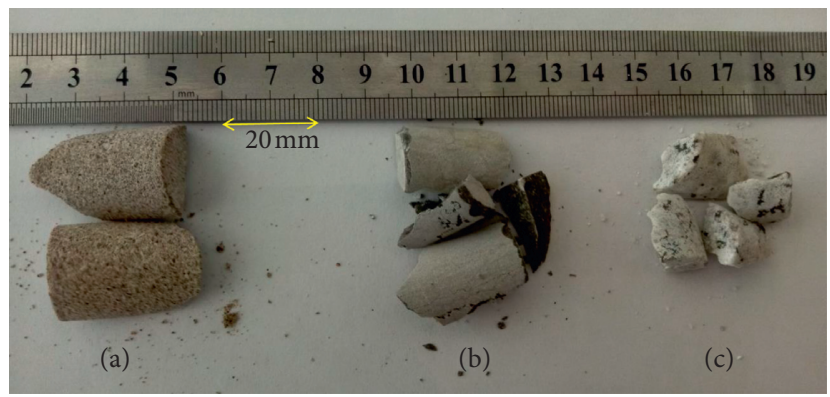

Figure 8: Debris of core columns under static-pressure breaking: (a) sandstone; (b) limestone; (c) granite.

concluded that the breaking work ratio of the core column is far less than that of the flat rock. The sensitivity of the 3 types of rock to the shape of the rock is different, and the granite is the most sensitive, followed by sandstone and limestone.

Figure 16 shows that the breaking work ratio of limestone decreases with the increase of the height of the core column, while the breaking work ratio of limestone and granite is discrete. The breaking work ratio of sandstone and granite is the minimum when the diameter of the core column is $35 \mathrm{~mm}$, and the breaking work ratio of sandstone and granite is the minimum when the diameter of the core column is $25 \mathrm{~mm}$. It can be concluded from Figure 16(b) that the breaking work ratio of the 3 rock samples is discrete, and the breaking work ratio of the limestone, sandstone, and granite reaches the minimum value when the diameters of the rock column are $40 \mathrm{~mm}, 30 \mathrm{~mm}$, and $30 \mathrm{~mm}$, respectively.

\section{Analysis of the Unit Experiment}

It is concluded from the unit breaking experiment that the breaking work ratio of the core column of the 3 types of rock is much less than that of the flat rock; it implies that a core column has been generated in the central area of the drill bit, 


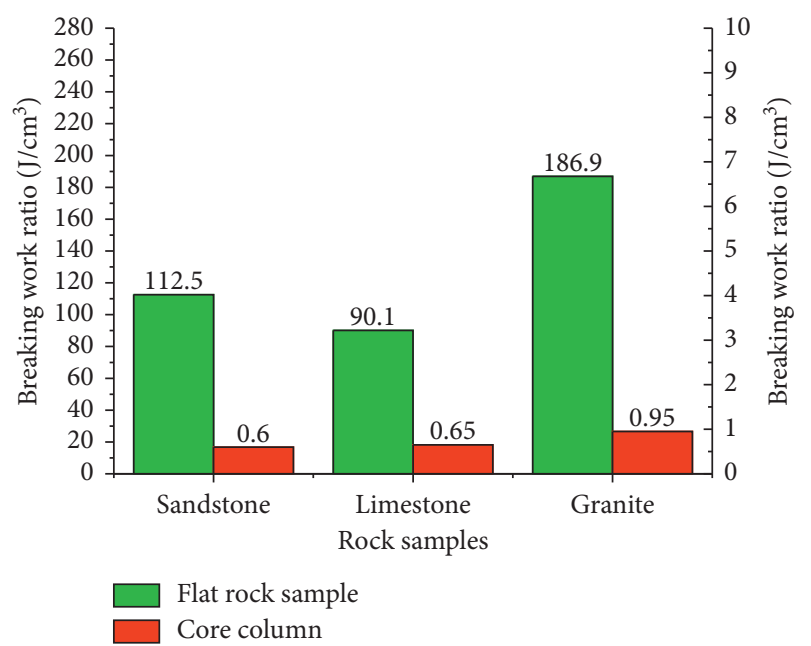

FIGURE 9: Breaking work ratio of flat rock samples and core columns in the static-pressure experiment.

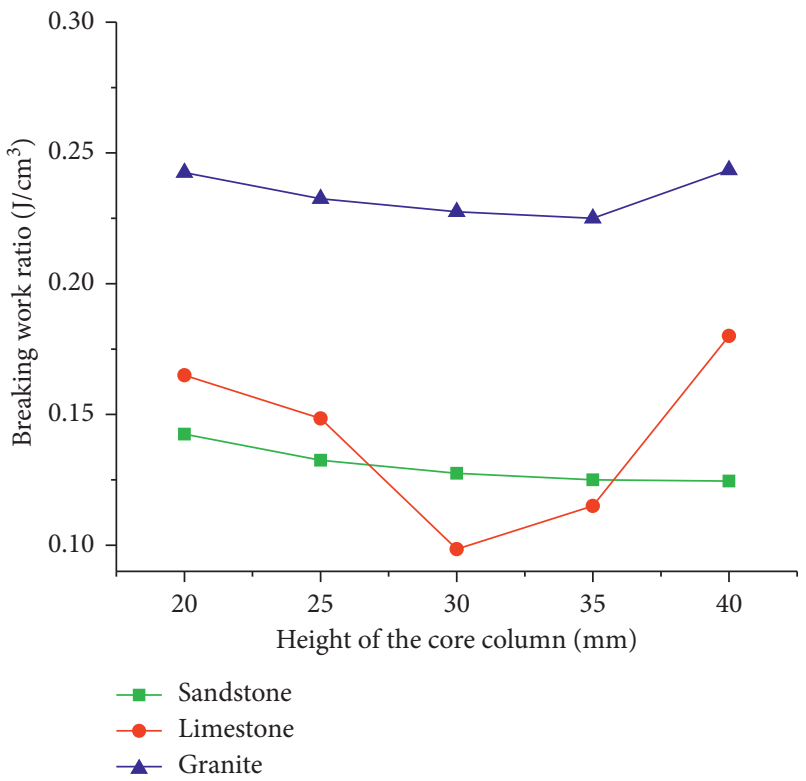

(a)

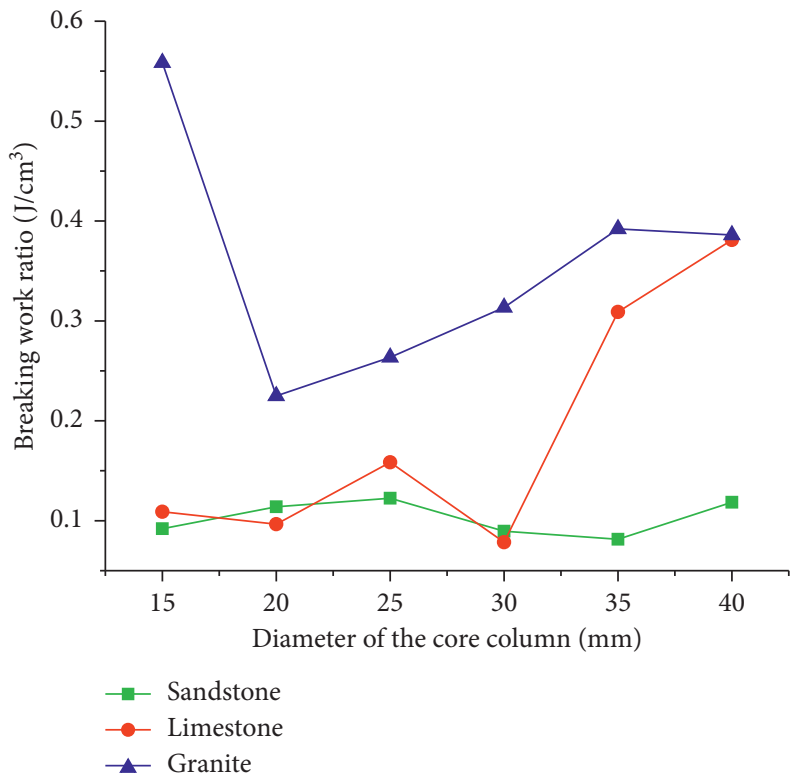

(b)

FIGURE 10: Effects of the breaking work ratio on the size of core columns in the static-pressure experiment.

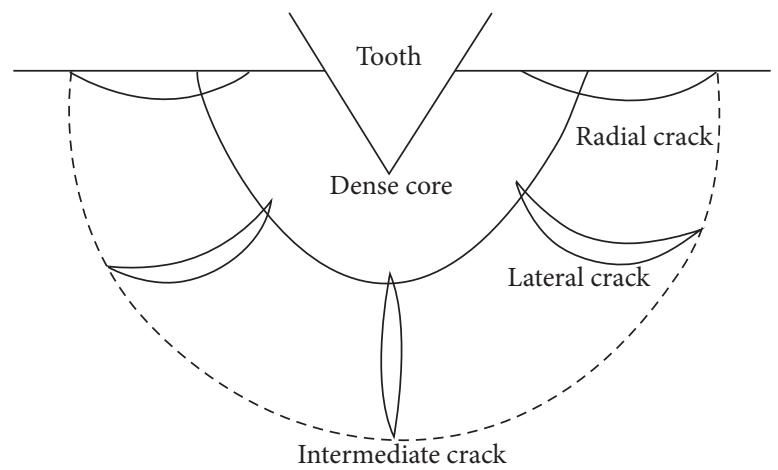

FIGURE 11: Graph for the tooth penetrating the rock. 


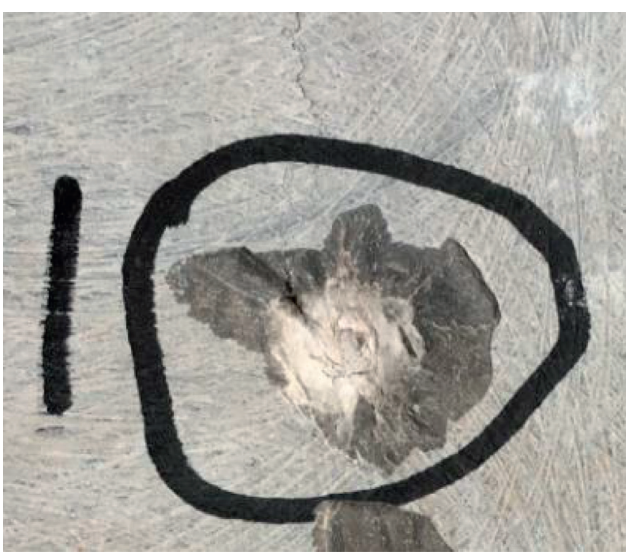

(a)

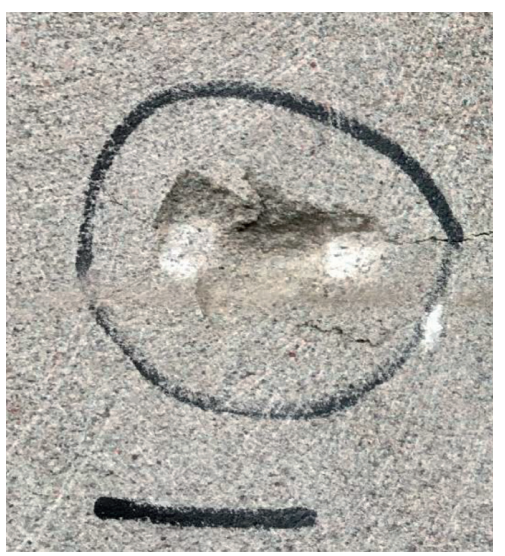

(b)

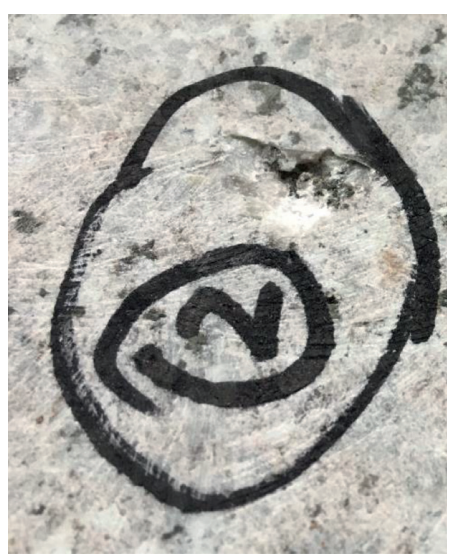

(c)

FIGURE 12: Breaking holes of the flat rock sample under impact: (a) sandstone; (b) limestone; (c) granite.

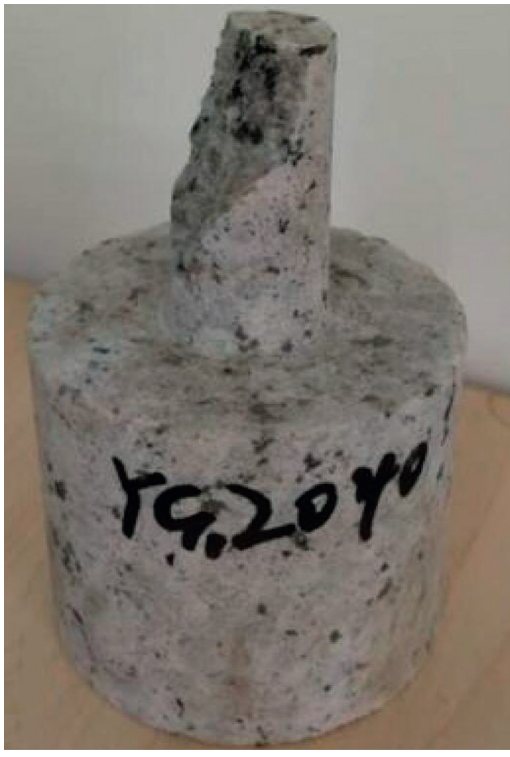

(a)

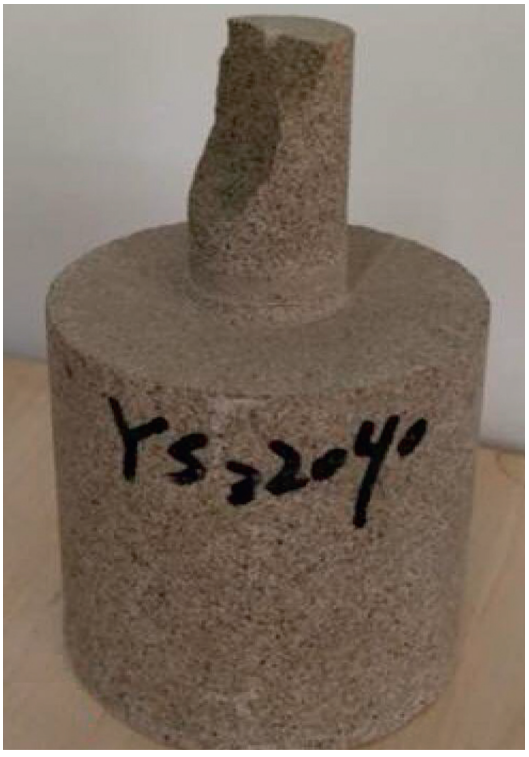

(b)

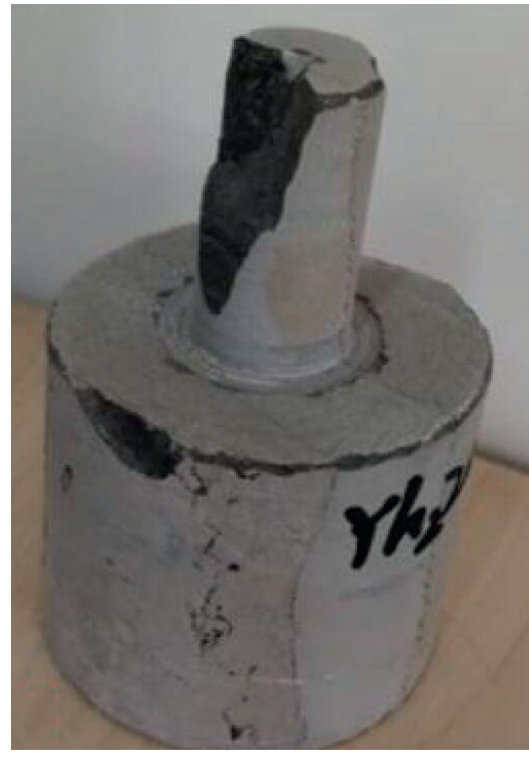

(c)

Figure 13: Core columns under impact: (a) sandstone; (b) limestone; (c) granite.

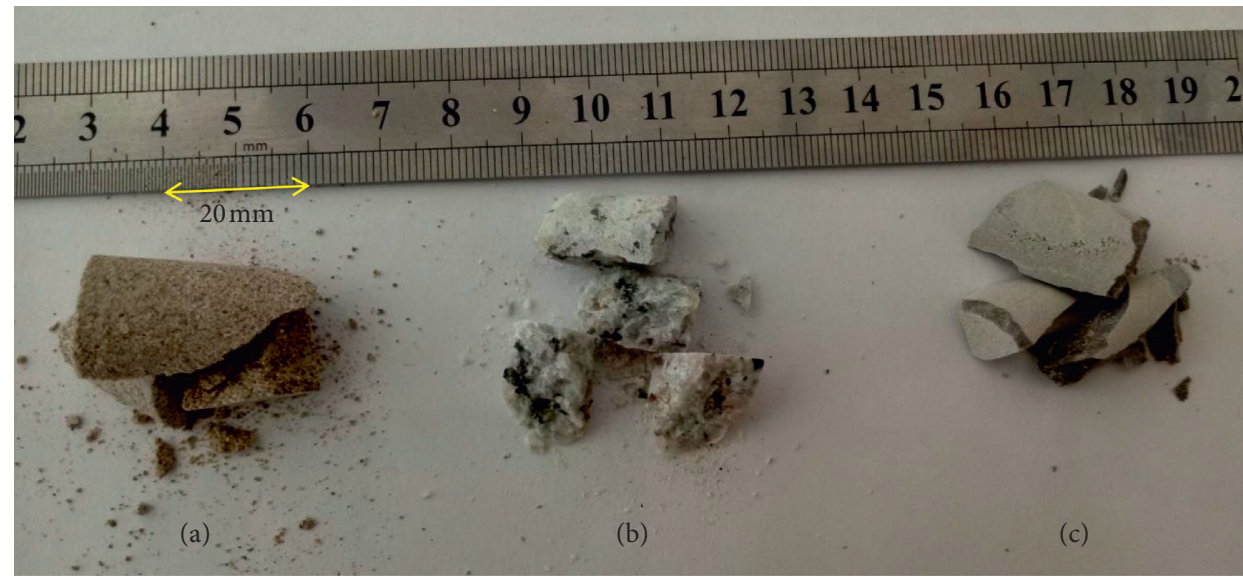

Figure 14: Debris of core columns under impact. 


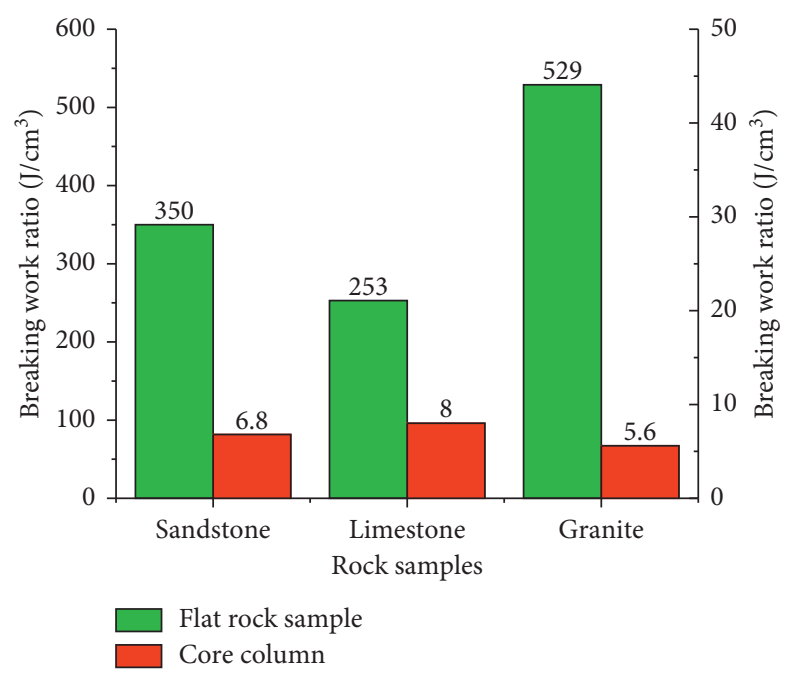

FIGURE 15: Breaking work ratio of flat rock samples and core columns in the impact experiment.

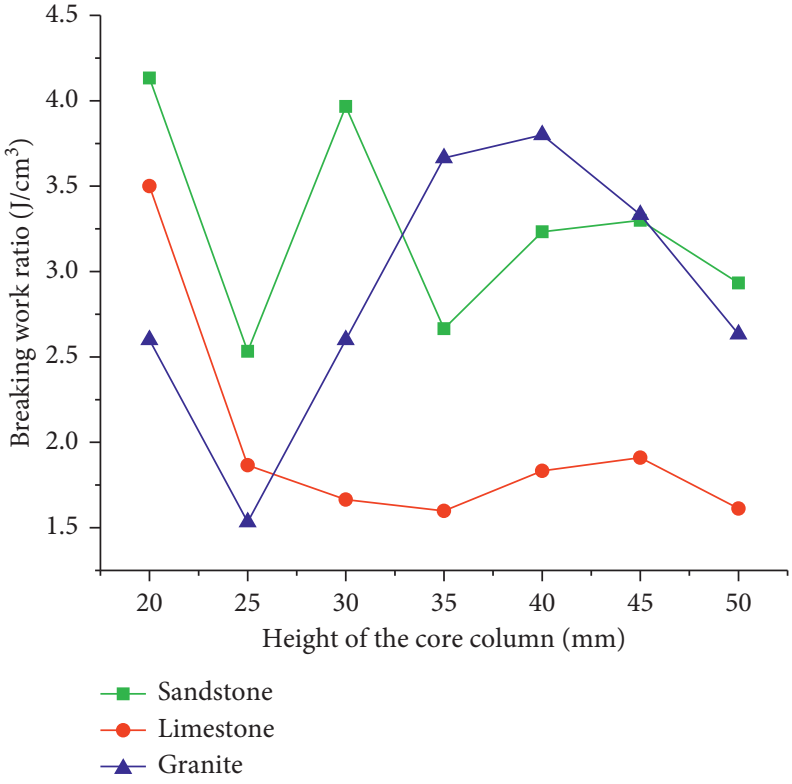

(a)

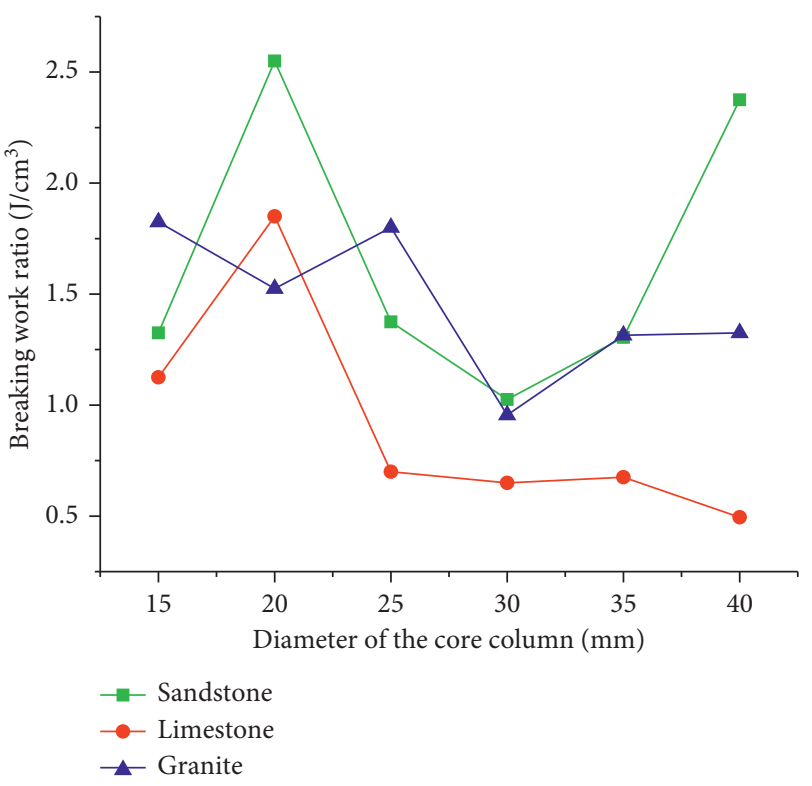

(b)

FigURE 16: Effects of the breaking work ratio on the size of core columns in the impact experiment.

which is beneficial to the volume breaking of the rock and can improve the rock-breaking efficiency of the drill bit, and the microcoring bit is suitable for raising speed in soft, medium, and hard formations. Figures 11 and 14 show that the size of debris particles obtained by static-pressure breaking is larger than that by the impact experiment. The breaking work ratio of the impact experiment is larger than that of the static-pressure breaking experiment which indicates that the rock-breaking efficiency of static-pressure breaking is higher than that of the impact experiment.

Through the unit experiment analysis, few differences between static-pressure breaking and impact are found: (1) The interaction time is different: the interaction time of static-pressure breaking is usually calculated in seconds, while that of the impact experiment is in microseconds $(\mu \mathrm{m})$; as for the static-pressure breaking experiment, the rock samples are often broken along a number of weak surfaces, and most of the rock samples are damaged locally when the impact load is loaded. Moreover, the dynamic strength of the rock sample is greater than the static strength. (2) Energy utilization is different: as for static-pressure breaking, the teeth directly contact rock samples and the energy loss is small, while during the impact experiment, some of the energy is converted into the kinetic energy of the teeth and the internal energy of the rock and the other part of the energy is dissipated. For instance, during the process of impact, the growth rate of internal cracks is relatively fast, so more energy is needed to increase the growth rate. 
Moreover, there are pores in the rock sample, which have a buffer effect on the impact force, thus increasing the impact energy required and reducing the energy utilization rate of the impact experiment. (3) The breaking work ratio is different: it is usually influenced by the mechanical properties of rock and the shape and the size of the teeth. The breaking work ratio of the impact experiment is greater than that of static-pressure breaking.

\section{Conclusion}

Through the unit experiment, some conclusions are obtained as follows:

(1) The breaking work ratio of the core column under static-pressure breaking and impact is much smaller than that of the flat rock sample. Static-pressure and impact have high rock breaking efficiency in sandstone, limestone, and granite, respectively.

(2) The breaking work ratio of the impact experiment is greater than that of static-pressure breaking. The height of the core column has little effect on the bit penetration rate, while core column diameter has greater effect on the bit penetration rate.

(3) A core column has been generated in the central area of the drill bit, which is beneficial to the volume breaking of the rock and can improve the rockbreaking efficiency of the drill bit, and the microcoring bit is suitable for raising speed in soft, medium, and hard formations and is helpful for geological logging.

(4) Through the experiment, the rock-breaking mechanism and accelerating mechanism of the microcoring PDC bits are obtained. When the core column is not formed at the bottom of the well, it is a special form with the highest rock-breaking efficiency for the microcoring PDC bits.

\section{Data Availability}

The experimental data used involve mechanical parameters of a new bit in this study, so they need to be kept confidential temporarily.

\section{Conflicts of Interest}

The authors declare that there are no conflicts of interest regarding the publication of this paper.

\section{Acknowledgments}

This work was supported by the Sichuan Science and Technology Program (Grant no. 2019YJ0537), Research and Field Test of High Temperature Geothermal Development Technology Program in Kenya (Grant no. 2016A-4801), China Postdoctoral Science Foundation (Grant no. 2019M653173), and National Natural Science Foundation of China (Grant no. 51504209).

\section{Supplementary Materials}

Static-pressure and impact experimental data. (Supplementary Materials)

\section{References}

[1] S. Dan, "A bit of History: Overcoming Early Setbacks, PDC Bits Now Drill 90\%-Plus of Worldwide Footage/Decades after Invention of Polycrystalline Diamond Cutters, PDC Bits Edge Out Roller Cones with Advances in Cutters, Stability, Hydraulics," Drilling Contractor, ADC, Eden Prairie, MN, USA, 2015.

[2] A. Rennie and P. Boonen, "An LWD tool suite for formation evaluation in HPHT environments," in Proceedings of the SPE Annual Technical Conference and Exhibition, pp. 11-14, ATCE, Anaheim, CA, USA, November 2007.

[3] A. Forsyth, B. Saucier, D. More, R. Stawaisz, D. Gill, and R. Younger, "The mechanical engineering challenges of designing HP/HT equipment," in Proceedings of the SPE Annual Technical Conference and Exhibition, pp. 11-14, ATCE, Anaheim, CA, USA, November 2007.

[4] M. Frydman, J. D. Restrepo, J. Palacio, C. Airoldi, and H. Eguia, "Reducing drilling risks in highly overpressurized formation: a case history in Nororiente Basin, Argentina," in Proceedings of the Latin America \& Caribbean Petroleum Enginering Conference, pp. 15-18, Buenos Aires, Argentina, April 2007.

[5] W. Meng and N. Prescott, "Dual subsea choke application for high pressure reservoir development," in Proceedings of the International Oil Conference and Exhibition in Mexico, pp. 27-30, Veracruz, Mexico, June 2007.

[6] B. Deschamps, S. Desmette, R. Delwiche et al., "Drilling to the extreme: the micro-coring bit concept," in Proceedings of the IADC/SPE Asia Pacific Drilling Technology Conference and Exhibition, pp. 25-27, Jakarta, Indonesia, August 2008.

[7] Y. Shinmoto, K. Wada, and E. Miyazaki, "Case study for core bit wear and its effect on core recovery and quality during deep-sea drilling expeditions," in Proceedings of the Brasil Offshore, pp. 14-17, Society of Petroleum Engineers, Macaé, Brazil, June 2011.

[8] H. Zhu, J. Shen, and F. Zhang, "A fracture conductivity model for channel fracturing and its implementation with discrete element method," Journal of Petroleum Science and Engineering, vol. 172, pp. 149-161, 2019.

[9] T. Y. Wang, "Principle and field application of micro core bit," Standard and Quality of China's Petroleum and Chemical Industry, vol. 23, pp. 78-80, 2017.

[10] H. C. Yu, J. Yang, R. D. Tao, and K. Z. Zhang, "Application of MICRO-CORE bit in Nanpu3-82well," West-China Exploration Engineering, vol. 12, pp. 66-68, 2016.

[11] S. Desmette, B. Deschamps, R. Birch, M. Naegel, and P. Essel, "Drilling hard and abrasive rock efficiently or generating quality cuttings? you no longer have to choose," in Proceedings of the SPE Annual Technical Conference, pp. 66-68, Denver, CO, USA, September 2008.

[12] Y. Shinmoto, S. Kodama, and B. Deschamps, "Evaluation of the micro-core quality using drilling mechanics data," in Proceedings of the International Symposium of the Society of Core Analysis, Society of Core Analysts, Scotland, UK, August 2012.

[13] Z. Maouche, F. AI-Rawahi, I. Agapie, M. Parasher, and T. AI Nahwi, "New PDC bit technology sets the standards in drilling hard and abrasive formations in Oman-case study," in 
Proceedings of the IADC/SPE Asia Pacific Drilling Technology Conference, pp. 25-27, Bangkok, Thailand, August 2014.

[14] K. Huang, Z. Ai, Y. Yang, and Z. Xie, "The improved rock breaking efficiency of an annular-groove PDC bit," Journal of Petroleum Science and Engineering, vol. 172, pp. 425-435, 2019.

[15] H. Zhu, J. Deng, X. Jin, L. Hu, and B. Luo, "Hydraulic fracture initiation and propagation from wellbore with oriented perforation," Rock Mechanics and Rock Engineering, vol. 48, no. 2, pp. 585-601, 2015.

[16] M. C. Zhang, R. Chen, S. W. Wang et al., "Application of micro core bit in Jidong oilfield," Liaoning Chemical Industry, vol. 45, pp. 124-126, 2016. 